\title{
WHAT IS THE TEMPERATURE DEPENDENCE OF THE CASIMIR FORCE BETWEEN REAL METALS?
}

\author{
G. L. KLIMCHITSKAYA* \\ Department of Physics, Federal University of Paraíba \\ Caixa Postal 5008, CEP 58059-970, João Pessoa, Pb-Brazil ${ }^{\dagger}$
}

\begin{abstract}
The situation with the temperature corrections to the Casimir force between real metals of finite conductivity is reported. It is shown that the plasma dielectric function is well adapted to the Lifshitz formula and leads to reasonable results for real conductors. The Drude dielectric function which describes media with dissipation is found not to belong to the application range of the Lifshitz formula at nonzero temperature. For Drude metals the special modification of the zero-frequency term of this formula is suggested. The contradictory results on the subject in recent literature are analysed and explained.
\end{abstract}

\section{Introduction}

Currently much attention is given to the Casimir effect and its topical applications in both fundamental physics and nanotechnology. This effect implies that there is some force acting between two uncharged bodies closely spaced in vacuum which is caused by zero-point oscillations of electromagnetic field. New precision measurements of the Casimir force have spurred the development of more exact theoretical methods taking into account such relevant factors as finite conductivity of the boundary material, nonzero temperature and surface roughness (for a review of modern experimental and theoretical developments in the Casimir effect see Ref. 2).

This paper is devoted to the investigation of the temperature dependence of the Casimir force acting between real metals, and one might believe that the question in the title has an apparent answer. There is the famous Lifshitz formula 3 in the form of a frequency sum for the Casimir force between two dielectric semispaces separated by a gap at nonzero temperature. Although there was a problem in application of this formula to planes made of ideal metals (the zero-frequency term becomes indefinite for the infinitely large dielectric permittivity), Schwinger, DeRaad and

*On leave from North-West Polytechnical University, St.Petersburg, Russia

$\dagger$ E-mail: galina@fisica.ufpb.br 
Milton 5 have long demonstrated how to proceed in the case of ideal metal. One must take the limit of infinite dielectric permittivity before putting frequency equal to zero. In doing so, the result for a perfect conductor is obtained from the Lifshitz theory. It is in agreement with the Casimir force calculated within the limits of quantum field theorywith Dirichlet boundary conditions in terms of the free energy density of vacuum $\mathbf{6}$. One would expect that in order to calculate the Casimir force between real metals it is suffice to substitute some model dependence of a metallic dielectric permittivity on frequency into the Lifshitz formula and make all summations and integrations correctly.

This approach, however, faces with serious problems. Starting in 2000, several theoretical groups undertook a number of studies of the Casimir force at nonzero temperature between real metals on the base of the Lifshitz formula. In Refs. 8,9 the Drude model was used to describe the dependence of the dielectric permittivity on frequency whereas in Ref. 10 the dielectric permittivity was described by the plasma model. In Refs. 11-13 both the plasma and Drude models were used on the base of the Lifshitz formula. The zero-frequency term of this formula was modified in Refs. 11-13 in the same way as for a perfect conductorl 5 . The results of Refs. 8,9 and 11-13 run into obstacles and are found to be in contradiction with the fundamental physical principles and experiment (see below). In Refs. 14-16 the Casimir force was also computed by the use of both plasma and Drude models. The results obtained in framework of the plasma model were found to be in agreement with those of Ref. 10. As for the Drude model, the pew prescription for the zerofrequency term of the Lifshitz formula was proposed 15 which makes it possible to avoid the nonphysical results of Refs. 8, 9, 11-13.

In this paper we report the present situation in the problem of calculation of the Casimir force between real metals at nonzero temperature. It is shown that in Refs. 8, 9 the Lifshitz formula was applied outside of its application range, whereas in Refs. 11-13 the unjustified prescription for the zero-frequency term of this formula was used. In Sec. 2 the brief formulation of the Lifshitz formula is presented.

Sec. 3 contains discussion of the application range of the Lifshitz formula starting from the modern derivation in the framework of Quantum Field Theory at nonzero temperature in Matsubara formulation. In Sec. 4 the results obtained in the framework of the plasma model are presented. Sec. 5 is devoted to the case of Drude model. In Sec. 6 the reader will find conclusions.

\section{The Lifshitz formula}

The original Lifshitz formula describing the Casimir and van der Waals force acting between two semispaces with a dielectric permittivity $\varepsilon(\omega)$ at a temperature $T$ separated by a gap of width a can be represented in the form of the sum over discrete frequencies

$$
F_{s s}(a)=-\frac{k_{B} T}{2 \pi} \sum_{l=-\infty}^{\infty} \int_{0}^{\infty} k_{\perp} d k_{\perp} q_{l}\left\{\left[r_{1}^{-2}\left(\xi_{l}, k_{\perp}\right) e^{2 a q_{l}}-1\right]^{-1}\right.
$$




$$
\left.+\left[r_{2}^{-2}\left(\xi_{l}, k_{\perp}\right) e^{2 a q_{l}}-1\right]^{-1}\right\}
$$

where $r_{1,2}$ are the reflection coefficients with parallel (perpendicular) polarization, respectively, given by

$$
r_{1}^{-2}\left(\xi_{l}, k_{\perp}\right)=\left[\frac{\varepsilon\left(i \xi_{l}\right) q_{l}+k_{l}}{\varepsilon\left(i \xi_{l}\right) q_{l}-k_{l}}\right]^{2}, \quad r_{2}^{-2}\left(\xi_{l}, k_{\perp}\right)=\left(\frac{q_{l}+k_{l}}{q_{l}-k_{l}}\right)^{2} .
$$

Here $\boldsymbol{k}_{\perp}$ is the momentum component lying in the boundary planes, $k_{\perp}=\left|\boldsymbol{k}_{\perp}\right|$, $\omega=i \xi$, and the following notations are used

$$
q_{l}=\sqrt{\frac{\xi_{l}^{2}}{c^{2}}+k_{\perp}^{2}}, \quad k_{l}=\sqrt{\varepsilon\left(i \xi_{l}\right) \frac{\xi_{l}^{2}}{c^{2}}+k_{\perp}^{2}}, \quad \xi_{l}=\frac{2 \pi l}{\beta}, \quad \beta \equiv \frac{\hbar}{k_{B} T},
$$

$k_{B}$ being the Boltzmann constant.

In the limit of zero temperature the Lifshitz formula can be written in terms of integrals

$$
\begin{gathered}
F_{s s}(a)=-\frac{\hbar}{2 \pi^{2}} \int_{0}^{\infty} d \xi \int_{0}^{\infty} k_{\perp} d k_{\perp} q\left\{\left[r_{1}^{-2}\left(\xi, k_{\perp}\right) e^{2 a q}-1\right]^{-1}\right. \\
\left.+\left[r_{2}^{-2}\left(\xi, k_{\perp}\right) e^{2 a q}-1\right]^{-1}\right\} .
\end{gathered}
$$

It is significant that both Eqs. (11) and (田) were originally derived for the case of nondissipative dielectric media. As was shown later 17.28 by the consideration of an auxiliary electrodynamic problem, at zero temperature the Lifshitz formula given by Eq. (4) preserves its validity even for media with dissipation (see Ref. 19 for details). Below we will make sure that this important conclusion can not be extended for the case of nonzero temperature. As a result, the Lifshitz formula (1) at $T>0$ can not be applied in the case of dissipative media without the appropriate modification of its zero-frequency term (see also Ref. 20)..

\section{Application range of the Lifshitz formula}

Recently the new derivation of the Lifshitz formula was performed 1 in the framework of Quantum Field Theory at nonzero temperature in Matsubara formulation. In this approach one considers Euclidean field theory with the electromagnetic field periodic in the Euclidean time variable within the interval $\beta$. For two semispaces the calculation of the free energy density is reduced to the solution of a one-dimensional scattering problem. The result is 3

$$
E_{s s}(a)=-\frac{\hbar}{2 \beta} \sum_{l} \int \frac{d \boldsymbol{k}_{\perp}}{(2 \pi)^{2}}\left[\ln s_{11}^{\|}\left(i \xi_{l}, \boldsymbol{k}_{\perp}\right)+\ln s_{11}^{\perp}\left(i \xi_{l}, \boldsymbol{k}_{\perp}\right)\right]
$$

where $s_{11}^{\|}\left(i \xi_{l}, \boldsymbol{k}_{\perp}\right)$ and $s_{11}^{\perp}\left(i \xi_{l}, \boldsymbol{k}_{\perp}\right)$ are the scattering coefficients for parallel and perpendicular polarizations, respectively. The solution of the scattering problem 
reads

$$
\begin{aligned}
& s_{11}^{\|}=\frac{4 \varepsilon\left(i \xi_{l}\right) k_{l} q_{l} e^{k_{l} a}}{\left[\varepsilon\left(i \xi_{l}\right) q_{l}+k_{l}\right]^{2} e^{q_{l} a}-\left[\varepsilon\left(i \xi_{l}\right) q_{l}-k_{l}\right]^{2} e^{-q_{l} a}}, \\
& s_{11}^{\perp}=\frac{4 k_{l} q_{l} e^{k_{l} a}}{\left(q_{l}+k_{l}\right)^{2} e^{q_{l} a}-\left(q_{l}-k_{l}\right)^{2} e^{-q_{l} a}},
\end{aligned}
$$

which is valid only if $q_{l} \neq k_{l}$ (see Eq. (3) for notations). If, however, $q_{l}=k_{l}$ the element of the scattering matrix $s_{11}^{\perp}$ proves to be arbitrary and the non-diagonal element $s_{12}^{\perp}=0$. What this means is that the scattering problem may not have any definite solution in the case when $q_{l}=k_{l}$. As discussed below, this fact is of crucial importance for the determination of the application range of the Lifshitz formula.

If $q_{l} \neq k_{l}$ and the scattering problem has definite solution, one can substitute Eq. (6) into Eq. (5) in order to get the free energy density. After performing renormalization, which is equivalent to the omitting of the free energy in the case of infinitely remote plates, one obtains

$$
\begin{gathered}
E_{s s}(a)=\frac{k_{B} T}{4 \pi} \sum_{l} \int_{0}^{\infty} k_{\perp} d k_{\perp}\left\{\ln \left[1-r_{1}^{2}\left(\xi_{l}, k_{\perp}\right) e^{-2 a q_{l}}\right]\right. \\
\left.+\ln \left[1-r_{2}^{2}\left(\xi_{l}, k_{\perp}\right) e^{-2 a q_{l}}\right]\right\} .
\end{gathered}
$$

It is easily seen that the Lifshitz formula (1) is obtained from Eq. (7) as the minus derivative of (8) with respect to $a$. Note that according to the Proximity Force Theoremen the force acting between a semispace (plate) and a sphere (spherical lens) can be calculated approximately as

$$
F_{s l}(a)=2 \pi R E_{s s}(a) .
$$

To discuss the application range of Eqs. (1), (7) let us start from the plasma model representation for the dielectric permittivity

$$
\varepsilon(\omega)=1-\frac{\omega_{p}^{2}}{\omega^{2}}, \quad \varepsilon(i \xi)=1+\frac{\omega_{p}^{2}}{\xi^{2}},
$$

where $\omega_{p}$ is the plasma frequency. If to substitute Eq. (9) into Eq. (3) one makes sure that $q_{l} \neq k_{l}$ including the zero-frequency case where

$$
q_{0}=k_{\perp}, \quad k_{0}=\sqrt{\frac{\omega_{p}^{2}}{c^{2}}+k_{\perp}^{2}} .
$$

The respective reflection coefficients at zero frequency are

$$
r_{1}^{2}\left(0, k_{\perp}\right)=1, \quad r_{2}^{2}\left(0, k_{\perp}\right)=\left(\frac{k_{\perp}-\sqrt{k_{\perp}^{2}+\frac{\omega_{p}^{2}}{c^{2}}}}{k_{\perp}+\sqrt{k_{\perp}^{2}+\frac{\omega_{p}^{2}}{c^{2}}}}\right)^{2} .
$$


Note that the first of them is equal to the reflection coefficient of real photons at zero frequency in the case of metals and the second one is smaller than unity. It is seen that the plasma model belongs to the application range of the Lifshitz formula and no difficulties arise. In the case of $\omega_{p} \rightarrow \infty$ one obtains

$$
\lim _{\omega_{p} \rightarrow \infty} r_{1}^{2}\left(\xi_{l}, k_{\perp}\right)=\lim _{\omega_{p} \rightarrow \infty} r_{2}^{2}\left(\xi_{l}, k_{\perp}\right)=1,
$$

i.e. the limit of ideal metal as it should be expected from physical considerations. In the next section some calculational results obtained in the framework of the plasma model are presented.

It is appropriate at this point to consider the cases when $q_{l}=k_{l}$ and the scattering problem may not have definite solution. Unexpectedly, this is the case for the nondissipative dielectric media described by

$$
\varepsilon(\omega)=\varepsilon(i \xi)=\varepsilon_{0}=\text { const }
$$

where at zero frequency $q_{0}=k_{0}=k_{\perp}$. In spite of this, some additional considerations help to fix the solution of the scattering problem for dielectrics. By the use of the unitarity condition which is valid for nondissipative media one arrives at $\left|s_{11}^{\perp}\left(0, k_{\perp}\right)\right|=1$, and after the application of the dispersion relation 1 the phase of the scattering matrix element can be also fixed with the result $s_{11}^{\perp}\left(0, k_{\perp}\right)=1$. It is significant that exactly the same result is obtainable from the general solution (6) of the scattering problem in a formal limit $\xi \rightarrow 0$. Thus, dielectrics turned out to belong to the application range of the Lifshitz formula along with metals described by the plasma model.

The values of reflection coefficients for dielectrics are found from Eqs. (2), (13)

$$
r_{1}^{2}\left(0, k_{\perp}\right)=\left(\frac{\varepsilon_{0}-1}{\varepsilon_{0}+1}\right)^{2}, \quad r_{2}^{2}\left(0, k_{\perp}\right)=0 .
$$

It is notable that they do not coincide with the values of reflection coefficients for real photons given by

$$
r_{1(R)}^{2}=r_{2(R)}^{2} \equiv r_{(R)}^{2}=\left(\frac{\sqrt{\varepsilon_{0}}-1}{\sqrt{\varepsilon_{0}}+1}\right)^{2} .
$$

As is seen from (14), (15), $r_{1}>r_{(R)}$ and $r_{2}<r_{(R)}$. The computational results for dielectrics are given below in Sec. 5 .

The other possibility when $q_{l}=k_{l}$ and the scattering problem may not have a solution takes place for metals described by the Drude dielectric function

$$
\varepsilon(\omega)=1-\frac{\omega_{p}^{2}}{\omega(\omega+i \gamma)}, \quad \varepsilon(i \xi)=1+\frac{\omega_{p}^{2}}{\xi(\xi+\gamma)},
$$

where $\gamma$ is the relaxation frequency. In fact here at zero frequency $q_{0}=k_{0}=k_{\perp}$, i.e. the same as for dielectrics. 
In this case, however, the unitarity condition is absent because of nonzero dissipation. As a result there is no way to solve the scattering problem at zero frequency and the matrix element $s_{11}^{\perp}\left(0, k_{\perp}\right)$ and thereby the reflection coefficient $r_{2}\left(0, k_{\perp}\right)$ remain undetermined. The conclusion is that real metals described by the Drude dielectric function are outside of the application range of the Lifshitz formula as given by Eq. (何).

For this reason it is of dubious value to substitute the reflection coefficients at zero frequency

$$
r_{1}^{2}\left(0, k_{\perp}\right)=1, \quad r_{2}^{2}\left(0, k_{\perp}\right)=0,
$$

following from Eqs. (2), (16) in the case of the Drude model, into the Lifshitz formula, as was done in Refs. 8, 9. The temperature corrections to the Casimir and van der Waals force computed in such a way may be and really are in contradiction with both the most fundamental physical principles and also with experiment (see Sec. 5).

To compute the Casimir and van der Waals force at nonzero temperature for real metal as described by the Drude model, some modification of the zero-frequency term of the Lifshitz formula is required. Such modification was attempted in Refs. 11-13 where the following values of the reflection coefficients were postulated for both plasma and Drude models

$$
r_{1}^{2}\left(0, k_{\perp}\right)=r_{2}^{2}\left(0, k_{\perp}\right)=1
$$

as given by the perfect conductors. This prescription is also shown to be in contradiction with both the general principles of thermodynamics and with experiment (see Secs. 4,5). The more adequate prescription applicable in the case of the Drude model and leading to no changes in the case of the plasma model was proposed in Ref. 15 and is discussed in Sec. 5 .

\section{Temperature Casimir force in the plasma model}

As was discussed in the preceding section, for real metals, as described by the plasma model, the Lifshitz formula is well defined and does not need any modification. The results for the force per unit area and free energy density between two semispaces are given by Eqs. (11), (7), where the reflection coefficients are defined in (2) and dielectric permittivity - in (9). The force between a semispace and a sphere can be obtained from Eq. (8). The computations in the framework of the plasma model were performed in Refs. 10,14,16. In the case of low temperatures $\left(T \ll T_{\text {eff }}\right.$ where $\left.k_{B} T_{\text {eff }}=\hbar c /(2 a)\right)$, or, equivalently, small separations the result can be obtained perturbatively 4

$$
\begin{gathered}
E_{s s}(a) \approx-\frac{\pi^{2} \hbar c}{720 a^{3}}\left\{1+\frac{45 \zeta(3)}{\pi^{3}}\left(\frac{T}{T_{e f f}}\right)^{3}-\left(\frac{T}{T_{\text {eff }}}\right)^{4}\right. \\
\left.-4 \frac{\delta_{0}}{a}\left[1-\frac{45 \zeta(3)}{2 \pi^{3}}\left(\frac{T}{T_{\text {eff }}}\right)^{3}+\left(\frac{T}{T_{\text {eff }}}\right)^{4}\right]\right\},
\end{gathered}
$$


where $\delta_{0}=c / \omega_{p}$ is the effective penetration depth of electromagnetic zero-point oscillations into a metal.

For the force acting between two semispaces the result is 14

$$
F_{s s}(a) \approx-\frac{\pi^{2} \hbar c}{240 a^{4}}\left\{1+\frac{1}{3}\left(\frac{T}{T_{e f f}}\right)^{4}-\frac{16}{3} \frac{\delta_{0}}{a}\left[1-\frac{45 \zeta(3)}{8 \pi^{3}}\left(\frac{T}{T_{e f f}}\right)^{3}\right]\right\} .
$$

It is notable that at low temperatures (small separations) the temperature correction in Eqs. (19), (20) depends only on the third and fourth powers in $T / T_{\text {eff }}$. This is in contradiction with Refs. 11-13 where for real metals, as described by the plasma model, the linear in temperature correction arises at low temperatures. Evidently, linear temperature correction leads to nonzero value of entropy at zero temperature. This value depends on the parameters of the system like the plasma frequency and space separation and, thereby, is in contradiction with the third law

of thermodynamics (Nernst heat theorem). Because of this, prescription (18) used in Refs. 11-13 is unacceptable in the case of metals described by the plasma model.

In the case of high temperatures (large separations) the results for the free energy density and force obtained on the base of the Lifshitz formula and plasma model $\operatorname{are} 14$

$$
E_{s s}(a)=-\frac{k_{B} T}{8 \pi a^{2}} \zeta(3)\left(1-2 \frac{\delta_{0}}{a}\right), \quad F_{s s}(a)=-\frac{k_{B} T}{4 \pi a^{3}} \zeta(3)\left(1-3 \frac{\delta_{0}}{a}\right),
$$

where $\zeta(z)$ is Riemann zeta-function.

In contrast to Eq. (21), at large separations the results of Refs. 11-13 contain no finite conductivity corrections, i.e. are given by the first contributions in the right-hand sides of (21). By way of example, at $T=300 \mathrm{~K}$ the finite conductivity corrections computed on the basis of Refs. 11-13 become zero at separations $a \geq$ $5 \mu \mathrm{m}$ regardless of the quality of a metal under consideration which is a nonphysical property.

\section{Temperature Casimir force in the Drude model}

As was shown in Sec. 3, the Drude dielectric function, which describes media with dissipation, is outside of the application range of the Lifshitz formula (1). To substitute the Drude dielectric function into the Lifshitz formula the special prescription must be adopted redefining its zero-frequency term. This should be done by following the general physical requirements (like the laws of thermodynamics) and preserving the solid results obtained earlier in the limiting cases of ideal metal and plasma model. Prescription of this kind was suggested in Ref. 15. It can be formulated as follows.

The zero-frequency term of the Lifshitz formula (5) for the free energy density can be represented as

$$
E_{s s}^{(l=0)}(a)=\frac{k_{B} T}{16 \pi a^{2}} \int_{0}^{\infty} y d y\left\{\ln \left[1-r_{1}^{2}(0, y) e^{-y}\right]\right.
$$




$$
\left.+\ln \left[1-r_{2}^{2}(y, y) e^{-y}\right]-\int_{0}^{y} d x \frac{\partial}{\partial x} \ln \left[1-r_{2}^{2}(x, y) e^{-y}\right]\right\} .
$$

To obtain Eq. (22) the continuous frequency $\xi$ was introduced in (3) and then the dimensionless variables were considered

$$
x=2 a \frac{\xi}{a}, \quad y=2 a \sqrt{\frac{\xi^{2}}{c^{2}}+k_{\perp}^{2}} .
$$

The derivative with respect to $x$ in (22) is identically equal to zero in the case of the plasma model. In the case of the Drude model, however, this derivative was proved to be a discontinuous function of the relaxation parameter $\gamma .15$ As a result, the presence of this term in Eq. (22) renders it unsuitable for real metals described by the Drude model.

To improve the situation one may use the fact that

$$
\frac{\partial \ln \left[1-r_{2}^{2}(x, y) e^{-y}\right]}{\partial x} \sim \frac{1}{\sqrt{\varepsilon-1}} \rightarrow 0 \text { with } \varepsilon \rightarrow \infty .
$$

Due to this the discontinuous term can be deleted by the prescription of Ref. 5 . As a result, the modified zero-frequency term of the Lifshitz formula is given by

$$
\begin{aligned}
& E_{s s}^{(l=0)}(a)=\frac{k_{B} T}{16 \pi a^{2}} \int_{0}^{\infty} y d y\left\{\ln \left[1-r_{1}^{2}(0, y) e^{-y}\right]\right. \\
& \left.\quad+\ln \left[1-r_{2}^{2}(y, y) e^{-y}\right]\right\}
\end{aligned}
$$

with all the other terms the same as in Eq. (5).

By way of example, in Fig. 1 the relative temperature correction to the free energy density between plates is presented at $T_{0}=300 \mathrm{~K}$ (which is the same as the relative temperature correction to the force between a sphere and a plate)

$$
\delta_{T}\left(E_{s s}\right)=\delta_{T}\left(F_{s l}\right)=\frac{E_{s s}\left(a, T_{0}\right)-E_{s s}(a, 0)}{E_{s s}(a, 0)} .
$$

The results obtained by the Lifshitz formula with a modified zero-frequency term (25) are shown by curve $1\left(\omega_{p}=12.5 \mathrm{eV}, \gamma=0.063 \mathrm{eV}\right.$ as for $\left.A l\right)$, curve 2 is calculated for dielectrics with $\varepsilon_{0}=7$, the dashed curve is obtained in the framework of Drude model and unmodified Lifshitz formulal (i.e. by the use of Eq. (17)). Both curves 1 and 2 demonstrate reasonable behavior. As to the dashed curve, it clearly demonstrates negative temperature correction at small and moderate separations. At $a=1 \mu \mathrm{m}$ this correction corresponds to about $17 \%$ of the force which is in contradiction with experiment22. Note that the negative temperature corrections are unacceptable from the theoretical point of view because they imply that the entropy of a system of two plates is negative (in this case within a separation range $0<a<4 \mu \mathrm{m}$ ). At high temperatures the result of Refs. 8,9 demonstrates only one half of the asymptotic value for a perfect conductor irrespective of how high the conductivity of real metal is which is a nonphysical property. 


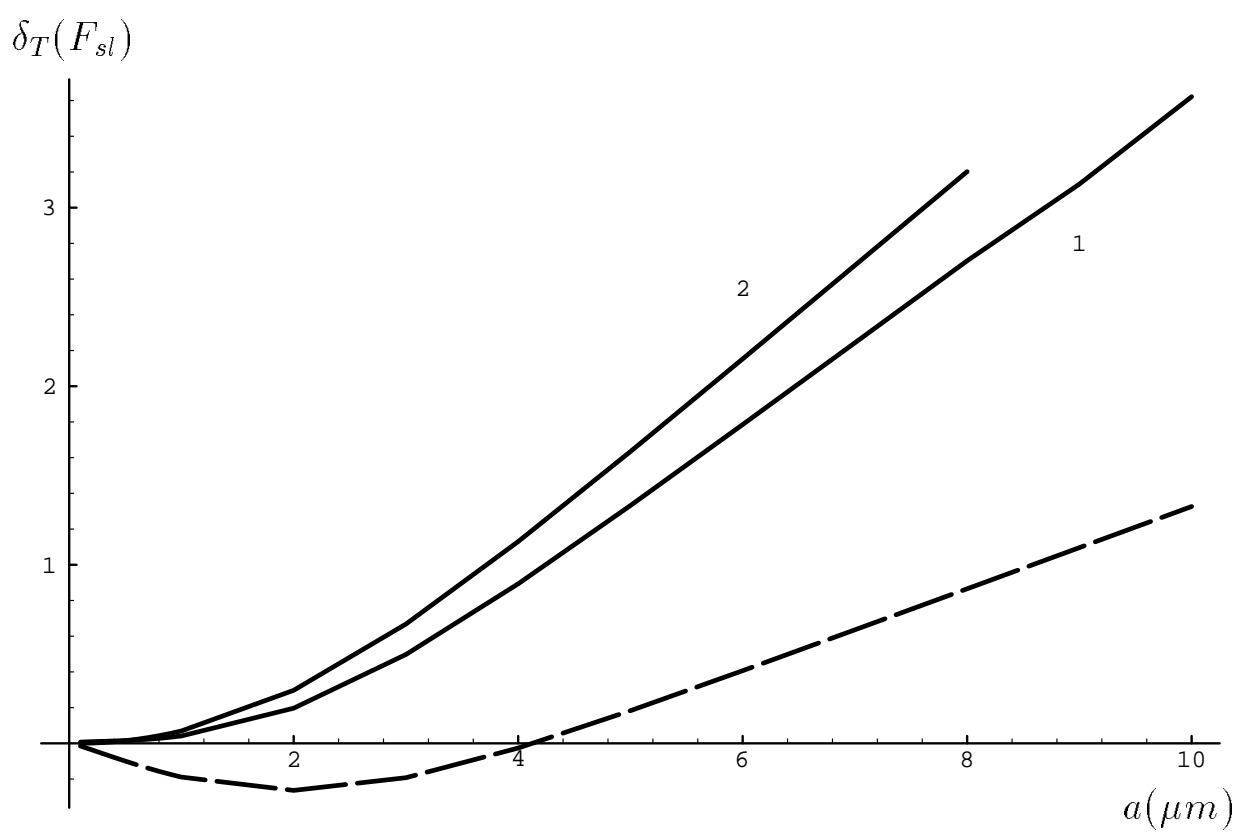

Fig. 1. Relative temperature correction to the Casimir force between a plate and a lens in dependence of separation. Curve 1 corresponds to Drude model (our computation), the dashed curve is obtained in Drude model with $r_{2}\left(0, k_{\perp}\right)=0$, and curve 2 is for the dielectric test bodies.

Analogical results are obtained for the force between two plates 15 . The negative temperature correction to the Casimir force obtained in Refs. 8, 9 is also in contradiction with the evident physical argument that with an increase of temperature the population of all modes, and thereby force modulus, increase.

The results obtained in Refs. 11-13 on the base of Drude model with a prescription (18) are also nonphysical. Here, once more, a linear (although positive) temperature correction arises at small separations. It is in contradiction with both the Nernst heat theorem and experiment23. At large separations the approach of Refs. 11-13 does not permit to describe the finite conductivity corrections to the Casimir force between real metals.

\section{Conclusions}

The following conclusions can be formulated from the above considerations.

1. The plasma model is well adapted for the description of the Casimir force between real metals at nonzero temperature. The Lifshitz formula with the plasma dielectric function is completely consistent mathematically and is not a subject for any modifications.

2. The Drude dielectric function describing the media with dissipation is outside 
of the application range of the Lifshitz formula at nonzero temperature. The zero frequency term of the Lifshitz formula with the Drude dielectric function remains indeterminate.

3. We propose the redefinition of the zero-frequency term of the Lifshitz formula in the presence of dissipation which is in accordance with the principles of thermodynamics and leads to physically consistent results.

4. The rigorous derivation of the Lifshitz formula at nonzero temperature in the case of dissipative media (including the zero-frequency term) is the important problem to be solved in near future.

\section{Acknowledgments}

The author is grateful to M. Bordag and V.M. Mostepanenko for helpful discussions. She is indebted to the Organizers of the Fifth Workshop on Quantum Field Theory under the Influence of External Conditions (Leipzig University, 2001) for kind hospitality. The financial support from CNPq is acknowledged.

\section{References}

1. H. B. G. Casimir, Proc. Kon. Nederl. Akad. Wet. 51, 793 (1948).

2. M. Bordag, U. Mohideen and V. M. Mostepanenko, Phys. Rep. 353, 1 (2001).

3. E. M. Lifshitz, Sov. Phys. JETP (USA) 2, 73 (1956).

4. E. M. Lifshitz and L. P. Pitaevskii, Statistical Physics, Part 2 (Pergamon Press, Oxford, 1980).

5. J. Schwinger, L. L. DeRaad, Jr. and K. A. Milton, Ann. Phys. (N. Y.) 115, 1 (1978).

6. J. Mehra, Physica 37, 145 (1967).

7. L. S. Brown and G. J. Maclay, Phys. Rev. 184, 1272 (1969).

8. M. Boström and Bo E. Sernelius, Phys. Rev. B61, 2204 (2000).

9. M. Boström and Bo E. Sernelius, Phys. Rev. Lett. 84, 4757 (2000).

10. C. Genet, A. Lambrecht and S. Reynaud, Phys. Rev. A62, 012110 (2000).

11. V. B. Svetovoy and M. V. Lokhanin, Mod. Phys. Lett. A15, 1013 (2000).

12. V. B. Svetovoy and M. V. Lokhanin, Mod. Phys. Lett. A15, 1437 (2000).

13. V. B. Svetovoy and M. V. Lokhanin, Phys. Lett. A280, 177 (2001).

14. M. Bordag, B. Geyer, G. L. Klimchitskaya and V. M. Mostepanenko, Phys. Rev. Lett. 85, 503 (2000).

15. G. L. Klimchitskaya and V. M. Mostepanenko, Phys. Rev. A63, 062108 (2001).

16. B. Geyer, G. L. Klimchitskaya and V. M. Mostepanenko, Int. J. Mod. Phys. A16, 3291 (2001).

17. Yu. S. Barash and V. L. Ginzburg, Sov. Phys. - Usp. (USA) 18, 305 (1975).

18. Yu. S. Barash and V. L. Ginzburg, Sov. Phys. - Usp. (USA) 27, 467 (1984).

19. V. M. Mostepanenko and N. N. Trunov, The Casimir Effect and Its Applications (Clarendon Press, Oxford, 1997).

20. R. Matloob and H. Falinejad, Phys. Rev. A64, 042102 (2001).

21. J. Blocki, J. Randrup, W. J. Swiatecki and C. F. Tsang, Ann. Phys. 105, 427 (1977).

22. S. K. Lamoreaux, Phys. Rev. Lett. 78, 5 (1997).

23. U. Mohideen and A. Roy, Phys. Rev. Lett. 81, 4549 (1998). 